\title{
Effect of sonication power on perfusion decellularization of cadaveric porcine kidney
}

\author{
Sreypich Say ${ }^{1}$, Nathaniel Dugos ${ }^{1 *}$, Susan Roces ${ }^{1}$ and John Martin Mondragon ${ }^{1}$ \\ ${ }^{1}$ Department of Chemical Engineering, Gokongwei College of Engineering, De La Salle University, 2401 Taft Avenue, \\ Manila 1004, Philippines
}

\begin{abstract}
Kidney problems rank 7th among the top 10 causes of mortality among Filipinos. One of the potential future treatment options is the use of bioengineered kidney. The preparation of scaffolds is the first step in kidney bioengineering and perfusion decellularization using chemicals is considered the most preferred preparation method to date. However, the use of chemicals alone requires long treatment time hence, in this study perfusion decellularization is to be enhanced by sonication treatment at varying sonication power. Decellularization was carried out by perfusing the kidney with $1 \%$ SDS and was subjected to sonication treatment with a $2 \mathrm{~h}$ sonication and $2 \mathrm{~h}$ rest cycle. The cycle is repeated until the kidney is clear and transparent. Washing using 1\% Triton X-100 and 1x PBS then follows to remove residual SDS. The extent of cell removal was determined by H\&E staining. The results showed that decellularization with sonication using $150 \mathrm{~W}, 200 \mathrm{~W}$ and $250 \mathrm{~W}$ required a treatment time of $24 \mathrm{~h}, 16 \mathrm{~h}$ and $12 \mathrm{~h}$ respectively compared to the $28 \mathrm{~h}$ treatment time of decellularization without sonication. The result clearly shows that with higher sonication power, the shorter is the decellularization time needed to prepare a good kidney scaffold.
\end{abstract}

\section{Introduction}

End-stage renal disease (ESRD) is a rising medical concern worldwide. According to the National Kidney and Transplant Institute (NKTI) in 2017, kidney disease ranks $7^{\text {th }}$ among other diseases leading to death among Filipinos [17]. The most common treatments are dialysis and transplantation but these are very costly and offers disadvantages. In the case of dialysis, it needs to be performed regularly [20] while shortage of organs available and the incompatibility of the kidneys are notable in transplantation treatments [9].

Tissue engineering and regenerative medicine provides a novel technique to solve ESRD by engineering a new kidney that is specific for each patient to avoid immunorejection and limited organ supply [7, 19]. The technique is composed of two stages which are the decellularization and recellularization process. Decellularization process is a technique used to remove the cellular content from the donor organ to minimize immunorejection $[\mathbf{1 9 , 2 3}]$. Meanwhile, recellularization is the process of repopulating the decellularized organ (scaffold) with patient stem cells [7, 24].

Decellularization may be done by chemical, physical and enzymatic methods [18]. Perfusion is the most common and preferred chemical method. Sodium dodecyl sulfate (SDS) was reported to be an effective chemical to remove cellular components. On the other hand, physical methods include sonication, freezing and agitation [12]. Enzymatic method makes use of Trypsin, Deoxyribonuclease (DNase) and Ribonuclease (RNase) * Corresponding author: nathaniel.dugos@dlsu.edu.ph
[8]. The successfully-prepared scaffold has to follow certain requirements such as preserved extracellular matrix (ECM), and completely or near completely removed cellular materials [6].

Decellularization of porcine kidney using chemical method alone requires a long time to remove cells, repeated treatment cycles and washing procedure $[10,13$, $15,21,25]$. To address this problem, the combination of chemical and physical method (sonication) was used in the study. This is due to the formation of cavitation bubbles during sonication that aids in the penetration of the chemical detergent by denaturing the cellular membrane resulting in the faster removal of cellular debris. Suitable sonication power is needed because too high sonication power might adversely damage the ECM structure while too low sonication power will not aid in cellular removal $[2-4,12,14,22,23]$.

The objective of this study was to investigate the effect of sonication power $(150 \mathrm{~W}, 200 \mathrm{~W}$ and $250 \mathrm{~W})$ on the decellularization of porcine kidney using Sodium dodecyl sulfate (SDS) with sonication. Remaining cellular content was analyzed after the decellularization process.

\section{Materials and methods}

\subsection{Perfusion decellularization system}

The perfusion decellularization of porcine kidney with sonication consisted of three beakers $(\mathrm{C}, \mathrm{G}, \mathrm{H})$ 
specifically for perfusion decellularization, chemical detergent, and waste solution, one peristatic pump (F), cooler bath $(\mathrm{E})$, sonicator $(\mathrm{A})$ and sonication probe horn (B) (See Fig. 1).

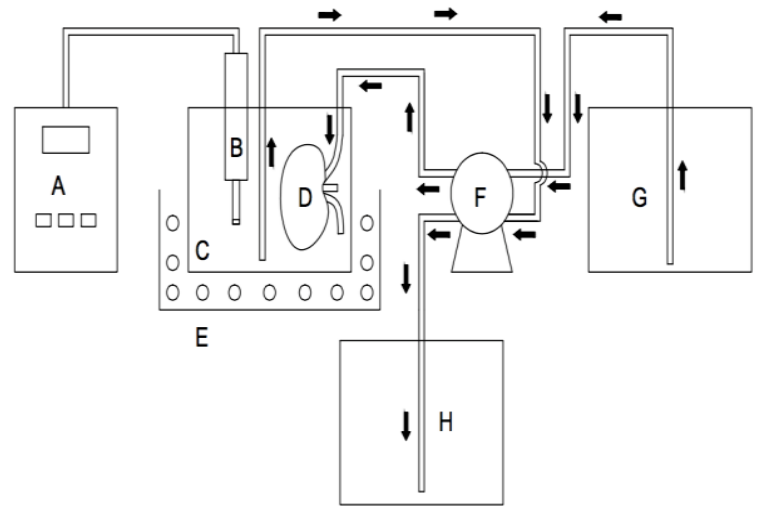

Fig. 1. Perfusion decellularization of porcine kidney with sonication setup.

Porcine female kidneys were supplied by Foremost Farms, Incorporated, Philippines. The porcine kidney was cannulated through its renal artery and then it was introduced to the perfusion decellularization system as shown in Figure 1. Distilled water was introduced through the renal artery for 3-5 hours at flow rate of $10 \mathrm{~mL} / \mathrm{min}$ to remove blood. Kidney is then sonicated with two hours on and two hours off cycle with a constant circulation of $1 \%$ Sodium dodecyl sulfate (SDS) at a flow rate of $10 \mathrm{~mL} / \mathrm{min}$. The cycle of sonication was repeated until the kidney turned white. The frequency was set at $20 \mathrm{kHz}$ and the power was varied from $150 \mathrm{~W}$, 200W and 250W. During sonication, the cooler bath was used to maintain the solution temperature. It was then washed with $1 \%$ Triton $\mathrm{X}-100$ for two hours at a flow rate of $10 \mathrm{~mL} / \mathrm{min}$ and $1 \mathrm{x}$ Phosphate buffered saline (1XPBS) for three hours at a flow rate of $15 \mathrm{~mL} / \mathrm{min}$. The product scaffold was stored in $10 \%$ formalin for further analysis. A control was performed following the protocol for decellularization of porcine kidney but without sonication.

Cellular content was analyzed using Haematoxylin and eosin $(\mathrm{H} \& \mathrm{E})$ staining.

\section{Results and discussion}

Decellularized scaffold was succesfully produced during the process as evident by the change in brown native color to white or transparent. Fig. 2 shows the native kidney lost its native cellular color after decellularization with respect to different sonication powers applied. The control (decellularization with 1\% SDS alone) set-up required 28 hours to completely remove cellular material. Meanwhile, it only took 24 hours, 16 hours and 12 hours for decellularization with sonication power of $150 \mathrm{~W}$, 200W and 250W respectively to decellularize the kidney. The faster removal of cellular material might be because of the cavitation bubbles formed during sonication which destroys cellular membrane and cellular components thus helping in the penetration of SDS. In addition, the number of cavitation bubbles increased with increasing sonication power thus leading to decreased decellularization time $[\mathbf{1 , 5}, \mathbf{1 1}, \mathbf{1 6}]$.
Fresh kidney

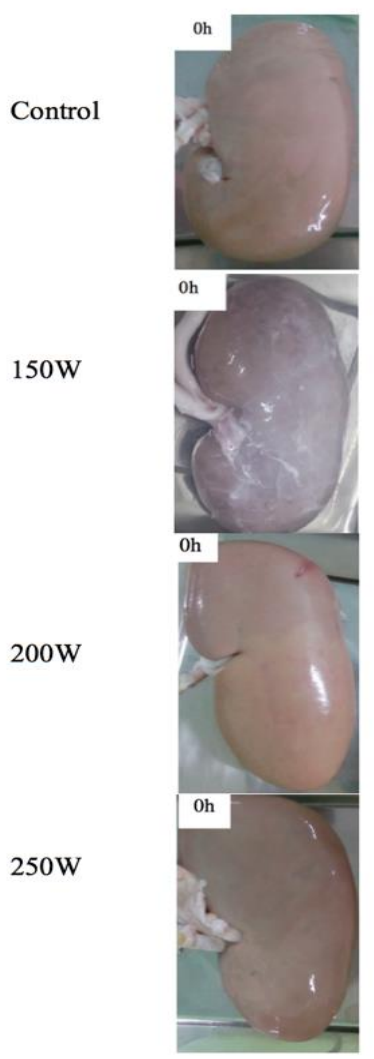

Final scaffold

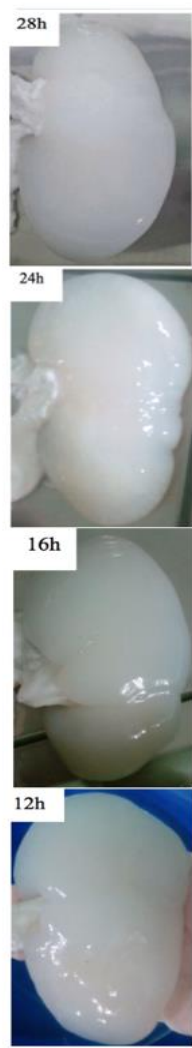

Fig. 2. Macroscopic evaluation of porcine kidneys before and after decellularization with varying sonication power applied and the control. The kidneys gradually turned translucent white with the progression of the decellularization process.
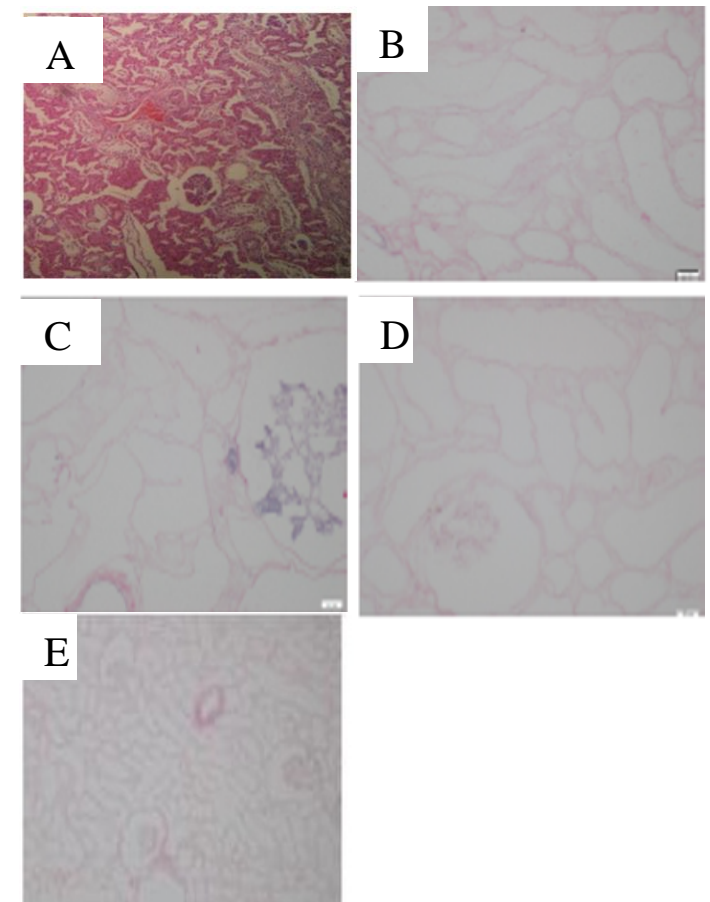

Fig. 3. Histological analysis of native (A), control (B), $150 \mathrm{~W}(\mathrm{C}), 200 \mathrm{~W}(\mathrm{D})$ and $250 \mathrm{~W}$ (E) through H\&E staining. The glomerular structure, tubular organization and the nucleus are clear. 
Fig. 3 shows the Hematoxylin and eosin (H\&E) stained images of native and decellularized kidneys. Percentage of cellular removal of native kidney, control (without sonication), $150 \mathrm{~W}, 200 \mathrm{~W}$ and $250 \mathrm{~W}$ were $0 \%$, $99 \%, 99 \%, 100 \%$ and $100 \%$ respectively as scored and evaluated by a pathologist.

Aside from the removal of cellular content, the preservation of various renal structures can also be observed by H\&E staining. The native ECM structure including glomerulus, tubular structure and blood vessels were preserved in the control, $150 \mathrm{~W}$ and $200 \mathrm{~W}$. Meanwhile in $250 \mathrm{~W}$, minimal damage can be observed due to the thinning of blood vessels and minimal disruption of glomerular basement membranes.

\section{Conclusion}

The results of the current study showed the successful decellularization of porcine kidneys with the aid of sonication. The resulting renal ECM scaffolds maintained their structure and appears devoid of cells. The best sonication power setting to achieve both $100 \%$ cellular removal and preservation of the ECM structure in the renal scaffold is $200 \mathrm{~W}$. This indicates that the combination of perfusion of $1 \%$ SDS with $200 \mathrm{~W}$ of sonication power in decellularization procedure could be of good choice for future studies due to its efficiency in cell removal while maintaining the native-like state of the ECM structure and with shorter decellularization time. In conclusion, sonication can be used to prepare decellularized scaffolds with the least amount of time. However, further studies need to be conducted to fully understand the effects of sonication in decellularization of whole organs such as the kidney.

The researchers would like to thank the following for financially supporting the project: ASEAN University Network/Southeast Asia Engineering Education Development Network (AUN/SEED-Net) and Commission on Higher Education Discovery-Applied Research and Extension for Trans/Inter-disciplinary Opportunities (CHED-DARETO) for their support in this project.

\section{References}

[1] Azhim, A., Ono, T., Fukui, Y., Morimoto, Y., Furukawa, K., \& Ushida, T. (2013). Preparation of Decellularized Meniscal Scaffolds Using Sonication Treatment for Tissue Engineering. 35th Annual International Conference of the IEEE EMBS, 69536956.

[2] Azhim, A., Shafiq, M., Rasyada, A. R., Furukawa, K., \& Ushida, T. (2015). The Impact of Acoustic Intensity on Solution Parameters and Decellularization Using Sonication Treatment. Journal of Biomaterials and Tissue Engineering, 5, 1-9.

[3] Azhim, A., Syazwani, N., Morimoto, Y., Furukawa, K., \& Ushida, T. (2014). The Use of Sonication Treatment to Decellularize Aortic Tissues for Preparation of Bioscaffolds. Journal of Biomaterials Applications, 29, 130-141.
[4] Azhim, A., Yamagami, K., Muramatsu, K., Morimoto, Y., \& Tanaka, M. (2011). The Use of Sonication Treatment to Completely Decellularize Blood Arteries: a Pilot Study. 2468-2471.

[5] Bob, S. (2013, December 5). Tovatech. Retrieved September 10, 2017, from http://www.tovatech.com/blog/255/ultrasoniccleaner/choose-right-power-rating- for-ultrasoniccleaner

[6] Crapo, P., Gilbert, T., \& Badylak, S. (2011). An Overview of Tissue and Whole Organ Decellularization Processes. National Institutes of Health, 32 (12), 3233- 3243.

[7] Gilpin, S. E., Guyette, J. P., Gonzalez, G., Ren, X., Asara, J. M., Mathisen, D. J., et al. (2014). Perfusion Decellularization of Human and Porcine Lungs: Bringing the Matrix to Clinical Scale. The Journal of Heart and Lung Transplantation, 33, 298-308.

[8] Gilpin, A., \& Yang, Y. (2017). Decellularization Strategies for Regenerative Medicine: From Processing Techniques to Applications. BioMed Research International, 2017, 1-13.

[9] Guan, Y., Liu, S., Liu, Y., Sun, C., Cheng, G., Luan, Y., et al. (2015). Porcine Kidneys as a Source of ECM Scaffold for Kidney Regeneration. Materials Science and Engineering C , 56, 451456.

[10] Hellstrom, M., El-Akouri, R., Sihlbom, C., Olsson, B., Lengqvist, J., Backdahl, H., et al. (2014). Towards the Development of a Bioengineered Uterus: Comparison of Different Protocols for Rat Uterus Decellularization. Acta Biomaterialia, 10, 5034-5042.

[11] Hesson, J. R. (2014). High Energy Sound Ultrasonics. Retrieved June 28, 2017, from http://www.hessonic.com/fundamentals-ofultrasonic-cleaning.html

[12] Hung, S. H., Su, C. H., Lee, F. P., \& Tseng, H. (2013). Larynx Decellularization: Combining Freeze-Drying and Sonication as an Effective Method. Journal of Voice, 27, 289-294.

[13] Liao, J., Joyce, E. M., \& Sacks, M. S. (2008). Effects of Decellularization on the Mechanical and Structural Properties of the Porcine Aortic Valve Leaflet. Biomaterials, 29, 1065-1074.

[14] Lin, C. H., Yang, J. R., Chiang, N. J., Ma, H., \& Tsay, R. Y. (2014). Evaluation of Decellularized Extracellular Matrix of Skeletal Muscle for Tissue Engineering. Int J Artif Organs, 34, 546-555.

[15] Meyer, S. R., Nagendran, J., Desai, L. S., Rayat, G. R., Churchill, T. A., Anderson, C. C., et al. (2005). Decellularization Reduces the Immune Response to Aortic Valve Allografts in the Rat. Evolving Technology, 130, 469-476.

[16] Moffa, T. (2010, Jan 12). Basic facts on Ultrasonic Cleaning, Process Description, the Ultrasonic Cleaning process. Retrieved Jun 28, 2017, from https://docmia.com/d/71980\#

[17] National Kidney and Transplant. (2017). National Kidney and Transplant Institute. Retrieved from 
http://www.nkti.gov.ph:

http://www.nkti.gov.ph/index.php/11-

services/654-kidney-transplantation\#

[18] Nonaka, P. N., Uriarte, J. J., Campillo, N., Melo, E., Navajas, D., Farre, R., et al. (2014). Mechanical Properties of Mouse Lungs Along Organ Decellularization by Sodium Dodecyl Sulfate. Respiratory Physiology \& Neurobiology.

[19] Park, K., \& Woo, H. (2012). Systemic Decellularization for Multi-Organ Scaffolds in Rats. Transplantation Proceedings, 44, 11511154.

[20] Raghavendra, Mallikarjun, \& Vidya M.J. (2013). Functions of Kidney \& Artificial Kidneys. International Journal of Innovative Research In Electrical, Electronics, Instrumentation And Control Engineering, 1, 1-5.

[21] Sullivan, D. C., Sani, S. H., Deegan, D. B., Baptista, P. M., Aboushwareb, T., Atala , A., et al. (2012). Decellularization Methods of Porcine Kidneys for Whole Organ Engineering Using a High-Throughput System. Biomaterials, 1-9.

[22] Syazwani, N., Azhim, A., Morimoto, Y., Furukawa, K., \& Ushida, T. (2014). Immune Response of Implanted Aortic Scaffolds Decellularized by Sonication Treatment. The 15th International Conference on Biomedical Engineering, IFMBE Proceedings, 43, 275-278.

[23] Syazwani, N., Azhim, A., Morimoto, Y., Furukawa, K., \& Ushida, T. (2015). Decellularization of Aorta Tissue Using Sonication Treatment as Potential Scaffold for Vascular Tissue Engineering. Taiwanese Society of Biomedical Engineering.

[24] Wang, Y., Bao, J., Wu, Q., Zhou, Y., Li, Y., Wu, X., et al. (2015). Method for Perfusion Decellularization of Porcine Whole Liver and Kidney for Use as a Scaffold for Clinical-Scale Bioengineering Engrafts. Xenotransplantation, 22, 48-61.

[25] White, L. J., Taylor, A. J., Faulk, D. M., Keane, T. J., Saldin, L. T., Reing, J. E., et al. (2016). The Impact of Detergents on the Tissue Decellularization Process: A ToF- SIMS Study. Acta Biomaterialia . 